III. Aus dem Dr. Senckenberg'schen Pathologischanatomischen Institut in Frankfurt a. M.

(Direktor: Geh. Med.-Rath Prof. Dr. Weigert.)

\title{
Ueber Fettfarbstoffe.
}

Von Dr. Gotthold Herxheimer, Assistenten am Institut.

Nachdem die Färbungsmethoden histologischer Präparate Allgemeingut der normalen und pathologischen Anatomen geworden, nachdem die grösstentheils empirisch gefundenen elektiven Färbungen ausserordentliche Früchte gezeitigt haben, versucht jetzt eine Anzahl Forscher auch die theoretische Farbchemie in die Histologie zu übertragen. Es ist dies um so verdienstlicher, als ja auch in der Industrie theoretisch und praktisch die Farbchemie einen ungeheuren Aufschwung genommen hat. Die grundlegende Eintheilung der in der histologischen Technik angewandten Farben ist die in basische und saure, sowie in durch Zusammentreten dieser beiden entstehende neutrale Farbstoffe. Die mittels dieser Eintheilung gewonnenen grossen Entdeckungen gehen bekanntlich auf Ehrlich, den Begründer der wissenschaftlichen Färbetechnik, zurück. Hierauf auch fusst der "Grundriss der Farbchemie" von Pappenheim. Eine Reihe von Azofarbstoffen nun, welche auf der einen Seite keine saure, auf der andern keine Basizität verleihende Gruppe enthalten, glaubt L. Michaelis besonders abtrennen zu können und bezeichnet sie als "indifferente“ Farbstoffe. In zwei Artikeln 1 ) beschäftigt sich der letztgenannte Autor des genaueren mit diesen und glaubt nachweisen zu können, dass eine solche Constitution gerade den und nur den Farbstoffen zukommt, welche Fett färben.

Ist dies von Michaelis gefundene Gesetz richtig, so ist es für Industrie wie Histologie von der grössten Bedeutung und ferner theoretisch, worauf auch Michaelis hinweist, von allergrösstem Interesse. Umsomehr glaube ich mit theoretischen Ueberlegungen und einigen praktischen Versuchen nicht zurückhalten zu dürfen, welche mir jene Michaelis'sche Lehre als noch nicht bewiesen erscheinen lassen.

Zu diesem Zwecke muss ich zunächst kurz den Gedankengang der Michaelis'schen Angaben referiren.

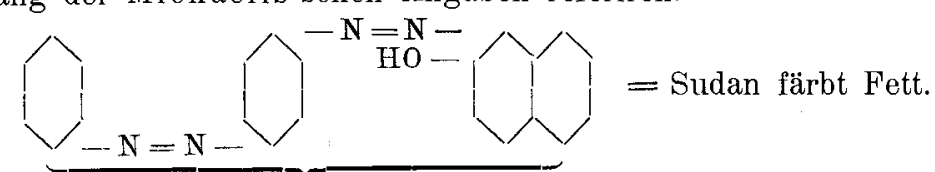

Azobenzolazo $\beta$ naphthol.

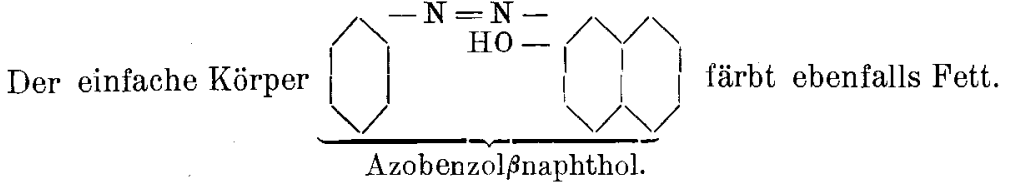

) Deutsche medizinische Wochenschrift 1901, No. 12. Virchow's Archiv 1901, Bd. CLXIV, Heft 2. 
Ebenso das von Michaelis in die histologische Technik eingefïhrte Fettponceau oder Scharlachroth

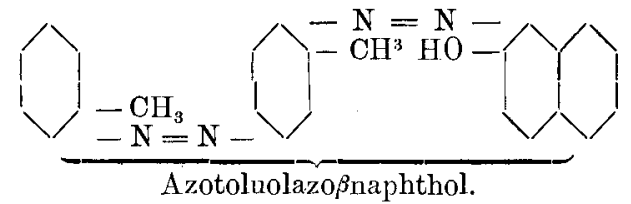

Allen diesen Farbstoffen ist die OH-Gruppe im Orthostellung zur Azogruppe zul eigen, und man könnte hierauf die Fettfärbung beziehen, zumal diese Farbstoffe mit aNaphthol, also mit der $\mathrm{OH}-$ Gruppe in Parastellung kein Fett färben. Die Orthostellung kann jedoch nicht das maassgebende sein, denn der Farbstoff

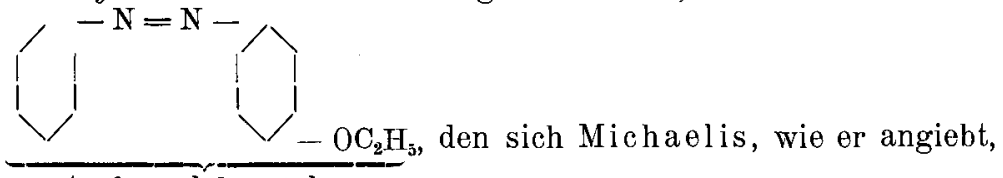

Azobenzolphenetol

herstellte, färbt ebenfalls Fett. In diesem Farbstoff ist ja gar keine OH-Gruppe vorhanden, und eben auf diesem Mangel der Hydroxylgruppe - so schliesst Michael is - scheint die Fähigkeit der Fettfärbung zu beruhen, denn auch jene obenerwähnten Fettfarbstoffe besitzen für gewöhnlich wahrscheinlich garnicht jene oben angegebene Formel mit der OH-Gruppe in Orthostellung zur Azogruppe, sondern sie sind so aufzufassen, dass das $H$ und $O$ getrennt ist. Also, um das einfachste Beispiel zu nehmen, von dem sich die anderen leicht ableiten lassen, statt

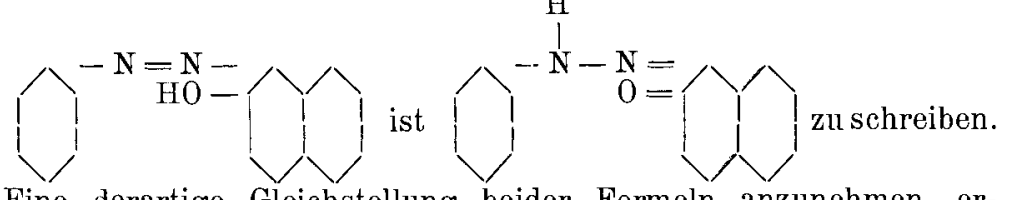

Eine derartige Gleichstellung beider Formeln anzunehmen, erlauben Analogieen mit den Versuchen von Zincke und Bindewald. Für die zweite Schreibweise spricht, dass phenolartige Verbindungen gewöhnlich mit Alkalien Salze bilden, jene Körper aber nicht. Für gewöhnlich also wäre die zweite Formel anzunehmen, in alkoholisch-alkalischer Lösung dagegen bilden jene Farbstoffe lösliche Salze: „In diesem Fall muss also eine Umlagerung in die erste Formel eintreten“. Und die das Facit ziehenden Sätze lauten: „Fettfarbstoffe sind diejenigen Azokörper, welche keine salzbildende Gruppe besitzen. Ich möchte diese Farbstoffe indifferente Farbstoffe nennen, im Gegensatz zu den sauren und basischen Farbstoffen." Und an anderer Stelle: „Es sind nämlich durchweg, und zwar nur sie (sc. die indifferenten Farbstoffe), Fettfarbstoffe."

Im Vorstehenden habe ich die Formeln etwas anders geschrieben als Michaelis, da sie nach dell Lehrbüchern so richtiger sind. Doch ändert dies ja an den Thatsachen garnichts. Gegen diesen Michaells'schen Gedankengang nun lässt sich folgendes einwenden. Da die alkoholische Lösung des Sudan oder Fettponceau mit Alkalien ein leicht lösliches Salz bildet, so sind wohl in der alkoholischen Lösung jene Farbstoffe der zweiten Formel entsprechend enthalten, also mit der OH-Gruppe am Naphtalinring. Und in alkoholischer Lösung wird ja das Sudan oder Fettponceau gerade zur Fettfärbung angewandt. Aber man könnte mir entgegenhalten, auch in der alkoholischen Lösung besteht die

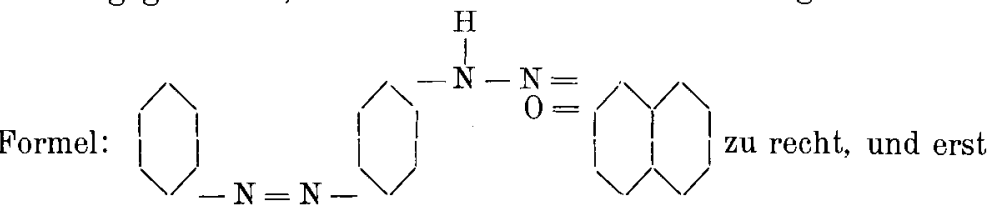

in der alkoholisch-alkalischen Lösung, in der sich das Sudan oder Fettponceau ja besonders leicht löst, bezw. ein leicht lösliches Salz bildet, tritt die Formel: ein. Hiervon schreibt Michaelis ja ausdrïcklich: „In diesem Fall muss also eine Umlagerung in die erste Formel eintreten." In Folge dessen stellte ich mir eine alkalisch-alkoholische Lösung von Sudan sowie Fettponceau her und versuchte damit Fett zu färben. Es handelte sich um eine Fettleber, und jede Verwechselung war ausgeschlossen. Auch hier erhielt ich das Fett sehr deutlich gefärbt, worauf ich unten zurückkomme. Also auch in einer Lösung des Sudan oder Fettponceau, in der nach Michaelis eigenen Schlussfolgerungen die $\mathrm{OH}-$, bezw. $\mathrm{ONa}-$ Gruppe vorhanden ist, lässt sich Fett färben; folglich kann die Fettfärbung nicht an den Mangel einer solchen Gruppe gebunden sein. Wollte man annehmen, der Körper lagere sich erst in dem Moment in die zweite Formel um, in dem das $\mathrm{H}$ durch das $\mathrm{Na}$ ersetzt wird, so ist auch dies kein Gegeneinwand; denn es handelt sich ja auch hier um das Salz eines sauren Farbstoffes, nicht um einen „indifferenten“ Farbstoff im Sinne Michaelis'.

Nach diesen theoretischen Schlüssen war es denn sofort wahrscheinlich, dass sich auch andere fettfärbende Azofarbstoffe finden liessen, welche eine sichere $\mathrm{OH}-G$ ruppe enthalten. Nimmt man einen Farbstoff, der zwei $\mathrm{OH}$ am Benzolring enthält, so könnte doch sicher nur die eine $\mathrm{OH}-G r u p p e$ der Annahme einer ganzen Anzahl chemischer Arbeiten entsprechend ${ }^{1}$ ) in $\mathrm{O}$ und $\mathrm{H}$ getrennt zu schreiben sein, die andere muss doch sicher als Hydroxylgruppe am Benzolring bleiben. Ein solcher Farbstoff ist das

Azobenzolresorzin mit der Formel:<smiles>CI1CC(O)CI(N=NC2CCCCC2)C1</smiles>

$\mathrm{OH}$

Dieser Farbstoff wird unter dem Namen „Cerasin Orange G“ von Cassella \& Co. in Frankfurt a. M. in den Handel gebracht. Der Farbstoff enthält beide OH-Gruppen wie das Resorzin, aus dem er hergestellt wird. Wollte man aber selbst annehmen, dass statt des $\mathrm{OH}$ der Orthostellung die Chinonstellung vorhanden ist, so muss sich die in Parastellung befindliche OH-Gruppe doch sicher in Phenolstellung befinden; denn einmal ist dies ja, wie soeben erwähnt, der Formel nach nöthig, und sodann bewahrt der Farbstoff vollkommen den phenolartigen Charakter. Er ist in Alkalien löslich, ebenso in Alkohol, in Wasser fast unlöslich. Dieses "Cerasin Orange G“ nun ist in der Technik als Fettfarbstoff bekannt, und auch histologisch färbt es Fett. Färbt sich hierbei auch das Grundgewebe leicht gelb-grünlich mit, so sticht doch das Fett durch seine gelbe bis orange Farbe deutlich hervor, besonders wenn man mit Hämatoxylin gegenfärbt. Zur Anwendung als histologisches Fettfärbmittel eignet sich das „Cerasin Orange G“ allerdings nicht, theils wegen seiner wenig intensiven Färbung, theils, da es allzu leicht aus der Lösung ausfällt und so Niederschläge verursacht. Aber wir haben hier einen sicher sauren Azofarbstoff, welcher Fett färbt.

Ich berücksichtigte bisher lediglich Azokörper, um zu zeigen, dass auch unter diesen solche, welche sicher nicht "indifferent" sind, im Stande sind, Fett zu färben. Versteht man also Michaelis' Arbeiten so, dass er sich lediglich mit den Azokörpern beschäftigt hat und unter diesen nur diejenigen für fettfärbend hält, welche eben „indifferent“ sind, so glaube ich dies in Vorstehendem an Azokörpern selbst widerlegt zu haben.

Die Thesen Mich aeli s' lassen nun aber weitergehende Schlussfolgerungen zu. Der Hauptsatz lautet in der Arbeit in Virchow's Archiv: „Fettfarbstoffe sind diejenigen Azokörper, welche keine salzbildende Gruppe besitzen". In der andern vorerwähnten Arbeit heisst es: „Aus dem Azobenzol kann nämlich auch durch Hinzutreten einer indifferenten Gruppe ein Farbstoff entstehen. Diese will ich als indifferente Farbstoffe zusammenfassen". Und weiter unten: „Es sind nämlich durchweg, und zwar nur sie (scil. die indifferenten Farbstoffe) Fettfarbstoffe". Man kann aus diesen Sätzen schliessen - und dem widerspricht anch sonst nichts in beiden Arbeiten -, dass Michaelis nur diejenigen Farbstoffe für histologisch fettfärbend hält, welche 1. Azokörper, 2. indifferent sind. Michaelis würde demnach annehmen, dass das, was er als „indifferent“ bezeichnet, nur unter den Azokörpern vorkommt, und ausserhalb dieser keinen fettfärbenden organischen Farbstoff kennen. Oder aber - und das glaube ich besonders der Arbeit in dieser Wochenschrift zu Folge - Michaelis hat die Azofarben nur als Beispiel genommen, um zu zeigen, wie sich indifferente Farbstoffe bilden, ebenso wie er an Azofarbstoffen als Beispiel kurz zuvor gezeigt hat, wie aus dem Chromogen saure und basische Farbstoffe entstehen. Würde Michaelis somit auch nicht leugnen, dass es ausserhalb der Azokörper noch organische Fett-

1) z. B. Liebermann, Berichte der Deutschen chemischen Gesellschaft Bd. XVI, S. 2858. Zincke und Bindewald, Berichte der Deutschen chemischen Gesellschaft Bd. XVII, S. 3026. Goldschmidt, Berichte der Deutschen chemischen Gesellschaft Bd. XXIV, S. 2300. 
farbstoffe giebt, so müssten doch auch diese ,indifferent" sein. Und in der That, warum sollte bei Azokörpern die Fettfärbung an eine bestimmte Gruppirung gebunden sein, bei andern Farbstoffen nicht? Es ist daher noch nöthig zu zeigen, dass es sichere Fettfarbstoffe giebt, welche keine Azokörper und nicht „indifferent" sind.

Hier wäre nun zunächst das auch von Michaelis erwähnte Alkannin zu nennen, welches sicher kein Azofarbstoff ist, da es gar keinen Stickstoff enthält; allein seine Constitution ist unbekannt. In einer glücklicheren Lage sind wir bei zwei Farbstoffen, auf welche ich von fachmännischer Seite aufmerksam gemacht wurde und welche beide einerseits keine Azokörper, andererseits ausgesprochen basisch sind. Es handelt sich zunächst um das Tetramethyldiamidoanthrachinon mit der Formel:<smiles>CN(C)C1CC2OC3CCCCC3OC3CC1CC2C(N(C)C)C3</smiles>

Ich wandte diesen Farbstoff in alkoholischer wie in alkalischalkoholischer Lösung an und erhielt eine sehr schöne Fettfärbung.

Der andere Farbstoff ist das Indophenol mit der Formel:<smiles>CN(C)C1CC[IH]C(N=C2CCC(=O)C3C[IH]CCC23)C[IH]C1</smiles>

Dieser Farbstoff färbt Fett ebenfalls, und zwar sehr deutlich, blau. Auf die Färbungen mit beiden Farbstoffen komme ich unten zuriick. Wir sehen also hier zwei Fettfarbstoffe auch ausserhalb der Azokörper, welche nicht ,indifferent" sind und doch Fett färben.

Durch seine theoretischen farbchemischen Annahmen nun kam Mich aelis dazu, neue Farbstoffe „synthetisch aufzubauen, welche das bisher gebräuchliche Sudan III an Färbeintensität übertrafen“, und unter diesen Farbstoffen schien ihm der von Kalle \& Co.

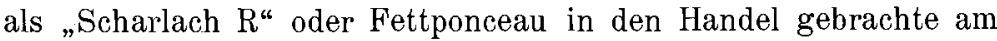
empfehlenswerthesten. Allerdings hätte Michaelis auf diesen Farbstoff auch ohne seine Fettfarbstofftheorie kommen können, und er ist von dieser völlig unabhängig, denn er hat dieselbe Constitution wie das Sudan III, nur dass er statt des Benzol Toluol, also die Methylgruppe, mehr enthält. Also schon der Formel nach ist es sehr wahrscheinlich, dass das Fettponceau ebenfalls Fett färbt, und zwar, der Vergrösserung des Moleküls entsprechend, etwas intensiver. Mit der Einführung dieses Farbstoffes aber hat sich Michaelis sicher ein Verdienst el'worben. Ich habe in der letzten Zeit viel mit "Scharlach R" gefärbt und mit Sudanfärbung verglichen. Bei gleicher Technik und Sicherheit schien auch mir das "Scharlach $R^{*}$ die viel schönere und deutlichere Färbung zu bieten.

Bei meinen obenerwähnten, zunächst aus theoretischen Gründen unternommenen Versuchen mit alkalisch-alkoholischen Lösungen nun beobachtete ich, dass sich auf diese Weise die Färbekraft beim Fettponceau wie beim Sudan erheblich steigern lässt. Hierauf, wie auf einige andere Punkte, die mir bei Fettfärbungen auch praktisch verwerthbar erscheinen, möchte ich noch mit einigen Worten eingehen. Giesst man einfach in eine gesättigte alkoholische „Scharlach R"-Lösung etwas Natronlauge, so wird die Lösung zwar dunkler, färbt jedoch, der Verdïnnung entsprechend, weniger intensiv. Eine entsprechende Menge Wasser zugesetzt, setzt die Tinktionskraft in Folge der Verdünnung ebenfalls herab, und zwar noch mehr, da etwas Farbstoff dabei ausfällt, von dem abfiltrirt werden muss. Da der Zusatz der Natronlauge zur alkoholischen Lösung aber die Lösungsfähigkeit steigert, so kann man noch Fettponceau in Substanzhinzusetzen. Filtrirt man nun ab, so ist diegesättigte alkalisch-alkoholische Lösung viel dunkler und färbt auch intensiver. Auf die Menge und Stärke der Natronlauge scheint nicht allzuviel dabei anzukommen. Hinzufïgen einer einprozentigen Lösung $20 \mathrm{zu} 80$ Alkohol steigert die Färbekraft schon, am besten bewährt aber hat sich mir folgende Lösung, bei der zugleich die durch Hinzusetzen der Natronlauge entstehende Verdünnung des Alkohols unter $70 \%$ vermieden ist:

Alcohol absolutus 70,0

Wasser 10,0

$10 \%$ Natronlauge 20,0

Hierin eine gesättigte Lösung des Fettponceau. Die eventuelle zerstörende Wirkung der Natronlauge auf das Gewebe wird hierbei wohl durch den starken Alkohol hintangehalten. Kann man auch nicht mit Hämatoxylin vorfärben, da die blaue Farbe in jener Fettponceaulösung wieder ausgezogen wird, so kann man doch die auf Fett mit obiger Lösung vorgefärbten Schnitte mit Hämatoxylin nachfärben, wobei die Kerne sich ebenso gut wie sonst färben. Auch Salzsäurealkohol zum Differenziren kann man hierbei anwenden. Diese alkoholisch-alkalische Lösung hat den grossen Vorzug, ausserordentlich viel schneller als die gewöhnliche alkoholische zu färben. 2-3 Minuten genügen im allgemeinen vollkommen. Auch an Intensität und Schönheit der Fettfärbung scheint mir keine der anderen von mir versuchten rothen Fettfarbstofflösungen ihr ebenbürtig zu sein. Am nächsten noch scheint mir das Tetramethyldiamidoanthrachinon zu kommen. Doch scheint mir diese Färbung nicht ganz so intensiv, und das Grundgewebe bleibt dabei nicht so rein weiss wie beim Fettponceau, ein Nachtheil, der sich allerdings durch Gegenfärben mit Hämatoxylin heben lässt. Ausserordentlich schön ist auch die blaue Fettfärbung, die ich mit Indophenol in gesättigter $70 \%$ alkoholischer Lösung bei einer Einwirkung von etwa 20 Minuten erhielt. Besonders schön ist diese Färbung bei Gegenfärbung mit Lithionkarmin Ist auch für gewöhnlich die Rothfärbung mit der alkalisch-alkoholischen Fettponceaulösung empfehlenswerther, so kann doch unter Umständen eine Blaufärbung des Fettes erwünscht sein, z. B. bei Lebern, bei denen hierbei der Gallenfarbstoff besser hervortritt.

Bei jenem oben aus theoretischen Gründen angeführten „Cerasin Orange G“, das ja leicht in Alkalien löslich ist, ist noch die Thatsache theoretisch interessant, dass eine solche wässerigalkalische Lösung absolut kein Fett färbt. Ein Diffundiren aus alkoholischer Lösung in das Gewebe ist offenbar, wie dies auch Michaelis annimmt, Vorbedingung für die histologische Fettfärbung. Nur ist, wie im Vorstehenden gezeigt, diese Fähigkeit nicht an „indifferente“ Farbstoffe (im Sinne Micha elis) gebunden. 